\title{
MONITORAMENTO E DIAGNÓSTICO DE MÚLTIPLOS SENSORES POR REDES NEURAIS AUTO-ASSOCIATIVAS
}

\author{
Javier Reyes* \\ javier.rys@gmail.com
}

\author{
Ricardo Tanscheit* \\ ricardodele.puc-rio.br \\ ${ }^{*}$ DEE-PUC-Rio \\ Rua Marquês de São Vicente, 225 \\ 22.451-900 Rio de Janeiro, RJ
}

\author{
Marley Vellasco* \\ marleydele.puc-rio.br
}


PALAVRAS-CHAVE: Redes neurais auto-associativas, sensores, manutenção, sistema de monitoramento de sinal.

\section{INTRODUÇÃO}

A automatização presente nas grandes empresas industriais de áreas como petróleo, mineração, gás, papel e celulose, água, etc. (Qiao et al., 2009; Eyng, 2008; Sanz et al., 2007; de Miguel and Blázquez, 2005; Böhme et al., 1999) está intimamente ligada a uma constante busca pela otimização dos processos de controle e monitoramento dos seus sistemas. A seleção, distribuição, instalação e controle da instrumentação são consideradas partes importantes e críticas na engenharia da empresa. Os atuais protocolos de barramento de campo, também chamadas redes industriais (Profibus, Modbus, Hart, ASI), permitem uma melhor comunicação e interação dos operadores e engenheiros com os equipamentos de campo, resultando em medidas confiáveis e informações de possíveis falhas nos sistemas (Tian et al., 2000). Não obstante, é comum que alguns sensores, às vezes os mais críticos, apresentem uma degradação não percebida pelo operador e levem a uma parada indesejada da produção (Afonso et al., 1998).

Nas últimas décadas têm sido desenvolvidas tecnologias que monitoram a condição dos processos industriais durante a operação da planta (Monsef et al., 2007). Com isto, as indústrias procuram substituir a manutenção preventiva por estratégias baseadas nas condições atuais (condition-based maintenance), obtendo potencialmente um método on-line mais eficiente. De especial interesse são as técnicas que controlam o estado dos sensores e os circuitos de instrumentação associados. Estas técnicas são comumente referidas como métodos de monitoramento on-line e são desenvolvidas para o monitoramento de sensores industriais e para a correção de medidas de um único sensor (Wrest et al., 1996; Xu et al., 1998; Hines et al., 2000).

Um problema comum enfrentado pelos projetistas de sistemas computacionais para acompanhar o desempenho de um processo dinâmico, com o objetivo de assegurar a operação dentro de limites impostos, é a confiabilidade do sensor. Como a saída dos sensores é a única fonte objetiva de informação para a tomada de decisão, é essencial que o estado de funcionamento destes sensores seja conhecido. Sensores inteligentes são capazes de enviar alertas para o controlador quando acontecem falhas na conexão, quedas de tensão nos terminais ou aumento da temperatura ambiente, mas não advertem sobre a ocorrência de uma degradação no seu estado físico, que pode causar inexatidão na grandeza medida. Os problemas de validação e substituição do sensor com falha devem ser considerados como parte integrante de um projeto moderno de monitoramento, de diagnóstico, ou de sistemas de controle (Tian et al., 2000).
A manutenção manual de sensores é realizada periodicamente, de forma a validar o funcionamento correto do instrumento. No entanto, dependendo da freqüência da manutenção, sensores defeituosos podem permanecer despercebidos por períodos longos de tempo. Desrespeitar os programas de manutenção pode trazer efeitos desfavoráveis para o processo, refletindo-se na qualidade do produto, causando perdas econômicas e, em muitos casos, acidentes, devido a um controle com sinais de realimentação imprecisos (Hines and Garvey, 2007). Por outro lado, a a manutenção manual é desnecessária em sensores que estejam funcionando corretamente, além de gerar um envelhecimento precoce dos componentes.

A Inteligência Computacional tem sido utilizada para desenvolver metodologias de detecção de falhas (Garcia-Alvarez et al., 2009; Kościelny and Syfert, 2006; Theilliol et al., 2002). Este método, em que um modelo computacional é projetado para predizer a saída do sistema real, não é adequado para executar um diagnóstico de sensores, uma vez que se baseia em dados de entrada (medições via sensores) corretos e pressupõe que as entradas para o sistema real e para o modelo estejam livres de falhas. Quando existe uma diferença notável entre a saída do sistema real e a saída do modelo, supõe-se que exista um problema no sistema real. No monitoramento e diagnóstico de sensores o foco é diferente, isto é, o objetivo é encontrar sensores disfuncionais (entradas do sistema) que provocam tais problemas (Najafi et al., 2004).

Em sistemas tradicionais de monitoramento e diagnóstico de falhas utiliza-se a chamada redundância por hardware, em que mais de um sensor realiza uma mesma medida. Embora esta técnica seja ainda utilizada por apresentar resultados confiáveis (Theilliol et al., 2002), ela apresenta algumas desvantagens, tais como o custo elevado de aquisição de um maior número de sensores ou a disponibilidade de espaço requerido para a instalação dos mesmos. Há também a chamada redundância por software, que faz uso da estimativa dos sinais por meio de um modelo matemático. Esta metodologia tem a principal vantagem de não implicar em um acréscimo de componentes ao sistema existente, reduzindo os custos e minimizando o espaço de instalação.

Sistemas como estes, cujo grau de complexidade é maior, têm sido pouco explorados no país. Os primeiros projetos de monitoramento de instrumentos de campo surgiram nos Estados Unidos em plantas de Energia Nuclear (Upadhyaya and Eryurek, 1992; Fantoni et al., 2003). No Brasil, as pesquisas concentraram-se em desenvolver modelos para autocorreção e auto-validação, utilizando técnicas como Lógica Fuzzy (Mendel, 1995) e Redes Neurais (Haykin, 1998), aplicadas ao monitoramento de um único sensor ou ao monitoramento da planta (Pereira et al., 2006; Bueno, 2006; Ga- 
lotto et al., 2007; Oliveira et al., 2009; Reyes et al., 2010). A utilização desses modelos em monitoramento on-line geraria uma complexidade maior do que a desejável, já que seria necessário projetar um sistema abrangendo distintos modelos para cada sensor em um mesmo processo. Uma metodologia que possa abranger um grupo grande de sensores ainda se faz necessária.

A motivação deste trabalho é desenvolver um modelo que possa realizar monitoramento on-line e auto-correção de medidas de múltiplos sensores, com o objetivo de reduzir os custos de manutenção e o risco de manter sensores defeituosos, aumentar a confiabilidade do instrumento e, consequentemente, reduzir o tempo de inatividade do equipamento. A capacidade das Redes Neurais (Haykin, 1998) de mapear sistemas complexos não lineares com múltiplas variáveis, sem necessariamente se conhecerem eventuais modelos matemáticos que descrevem seus comportamentos, é uma alternativa para realizar a verificação e o processamento de sinais dos instrumentos de medição.

O modelo proposto neste trabalho foi concebido a partir do estudo das características desejadas para o sistema de autocorreção e diagnóstico de sensores que operam em um processo contínuo. Optou-se por utilizar a arquitetura de Redes Neurais Auto-Associativas (Kramer, 1991), que permitem a filtragem de ruídos nas medidas, a auto-correção do sinal com falha quando o sensor apresenta um erro do tipo desvio (drift fault) - definido como uma baixa taxa de variação do valor esperado do sinal no tempo - e o tratamento de falhas grosseiras (gross faults) - definidas como falhas drásticas no sensor.

Este artigo contém cinco seções adicionais. A seção 2 apresenta a arquitetura de Redes Neurais Auto-Associativas (RNAA) proposta para a realização da auto-correção de medidas dos sensores, assim como a descrição do treinamento robusto para RNAA. A seção 3 apresenta o modelo de monitoramento on-line de instrumentos, constituído por um módulo de auto-correção, baseado no treinamento modificado da RNAA (RNAAM), e por num módulo de lógica de decisão, baseado no SPRT (Hines et al., 2000). A seção 4 apresenta o estudo de caso: sensores industriais de controle e monitoramento de um motor de combustão interna utilizado em um caminhão de mineração. Esta aplicação possui um grande número de variáveis de entrada (medidas dos sensores), que permite avaliar o desempenho do modelo proposto. Finalmente, a seção 5 conclui o trabalho.

\section{ARQUITETURA DE REDES NEURAIS AUTO-ASSOCIATIVAS}

As redes neurais auto-associativas são inspiradas na metodologia NLPCA (nonlinear principal components analysis)
(Torres et al., 2004; Cuenca et al., 2004). Simplificadamente, nesta metodologia, dados de entrada de um sistema são mapeados na saída por meio de uma função não linear G. A reconstrução do dados originais é efetuada por um "desmapeamento", expresso por uma função $\mathrm{H}$, também não linear. As funções $\mathrm{G}$ e H são escolhidas de forma a se minimizar a perda de informação no processo mapeamento-desmapeamento.

Tendo em vista uma representação desse processo por meio de uma rede neural, é importante recordar que redes neurais podem implementar uma vasta gama de funções nãolineares. Esta característica pode ser usada na construção de uma rede para obtenção de componentes principais nãolineares (Torres et al., 2004). Na arquitetura em questão, fazse uso de duas redes neurais conectadas em série, responsáveis pela implementação do mapeamento, $\mathrm{G}$, e do desmapeamento, H, dos sinais, conforme mostrado na Figura 1.

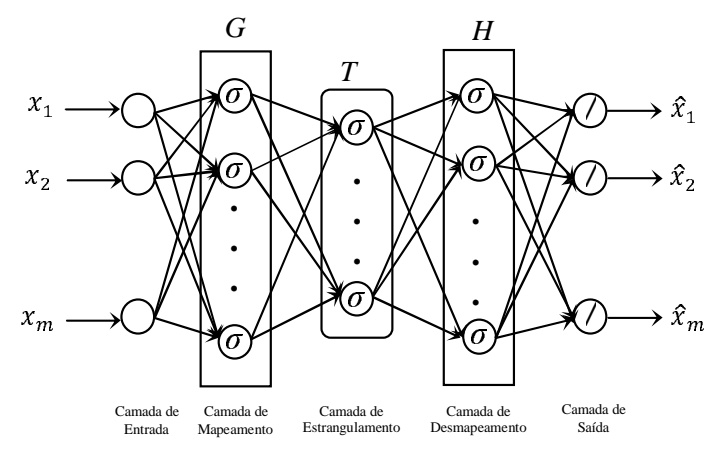

Figura 1: Rede neural auto-associativa.

As medidas dos sensores formam o vetor de entrada da rede, $\mathbf{X}$; em consequência, a dimensão $m$ da camada de entrada corresponde à dimensão do vetor de medidas. A camada de saída produz o vetor de dados reconstruído, de mesma dimensão da camada de entrada. A primeira camada escondida representa o mapeamento $\mathrm{G}$ e contém um número de neurônios maior do que número de entradas. A segunda camada escondida representa a camada de estrangulamento (bottleneck) $\mathrm{T}$, de dimensão inferior à da camada de entrada. A terceira camada escondida representa a camada de desmapeamento $\mathrm{H}$, de dimensão igual à da camada de mapeamento. Observe-se que a camada escondida T é a projeção do vetor de entrada no espaço de características. Nesta rede, os pesos são atualizados para cada amostra, de forma que, ao final do processo, a distribuição dos pesos possa caracterizar a rede neural como uma matriz identidade. Em outras palavras, busca-se otimizar a topologia da rede de forma a reconstruir as $m$ variáveis de entrada com o menor erro possível, utilizando-se para tal um conjunto reduzido de neurônios na camada de estrangulamento.

A camada de estrangulamento desempenha um papel chave na funcionalidade da rede auto-associativa, pois força uma 
codificação e compressão das entradas, com uma posterior decodificação ou descompressão. Eventuais correlações ocultas existentes nos dados serão capturadas pela camada de estrangulamento e os efeitos de não correlação - produzidos, por exemplo, por ruído nos sinais - serão excluídos nesta camada (Kramer, 1991). Se a rede é treinada com dados adequados, a saída para um novo dado da mesma distribuição do conjunto de treinamento estará o mais próximo possível do estado livre de ruído. Não existe um método para decidir o número de neurônios das camadas escondidas da RNAA. No caso das camadas de mapeamento e desmapeamento, devese adotar uma dimensão suficientemente grande para garantir que a rede consiga recuperar os sinais de entrada, sem risco de over-fitting. A validação cruzada também pode ser usada para selecionar o número apropriado de neurônios nestas camadas (Marseguerra and Zoia, 2005a; Marseguerra and Zoia, 2005b; Kramer, 1992).

\subsection{Treinamento Robusto}

O treinamento robusto (Kramer, 1992) é uma importante ferramenta para a solução de problemas com erros súbitos (desvio e offset) e ruído nas medidas. Fornece-se à rede neural um conjunto de dados em que alguns padrões, selecionados de forma aleatória, são corrompidos, forçando-se a rede a obter os resultados corretos (sem perturbações). Os padrões a serem corrompidos são escolhidos com distribuição de probabilidade uniforme do conjunto de treinamento e cada corrupção consiste em variar uma entrada selecionada aleatoriamente dentre as amostras, enquanto todas as outras entradas mantêm os seus valores corretos (Marseguerra and Zoia, 2005b).

Considerando uma variável de entrada $\mathbf{X}=\left[x_{1}, x_{2}, \ldots, x_{n}\right]$ com $n$ amostras, a uma amostra $j$ é agregado um ruído aleatório $\delta$, de maneira a formar a nova entrada $\mathbf{X}^{*}=$ $\left[x_{1}, x_{2}, \ldots, x_{j+\delta}, x_{j+1}, \ldots, x_{n}\right]$; a RNAA é forçada a fornecer como saída os dados originais de $\mathbf{X}$. Este procedimento é repetido várias vezes para as $m$ entradas da rede neural, de forma que todas as amostras do conjunto de treinamento sejam corrompidas em diversas ocasiões. Assim, o algoritmo de aprendizagem visa a sintonizar a rede para que as suas respostas estejam próximas dos valores desejados quando os sinais de entrada apresentam ruído ou erros súbitos.

A rede treinada desta forma consegue corrigir as medidas dos sensores quando somente um deles apresenta falhas; caso existam mais sensores defeituosos, ela não conseguirá corrigir os erros de forma adequada. Abordagens que procuram superar esta deficiência (Najafi et al., 2004; Singh, 2004; Marseguerra and Zoia, 2006; Antory et al., 2008) fazem uso de estruturas mais complexas ou de alto custo computacional, inviáveis para procedimentos on-line. O modelo proposto neste trabalho emprega somente uma rede neural para reconstruir os sinais dos sensores com falha, sem o auxílio de algoritmos adicionais ou de outra rede, simplificando, assim, o treinamento.

\section{MODELO DE MONITORAMENTO}

Os modelos de monitoramento podem ser divididos em duas grandes categorias: redundantes e não redundantes. Na modelagem redundante, um conjunto de sensores verifica o funcionamento de um mesmo processo. A média das leituras dos vários sensores pode ser utilizada, por exemplo, para se obter o resultado. Em contraste, as técnicas de modelagem não redundantes se baseiam na estimação das medidas de instrumentos correlacionados (Hines and Garvey, 2007).

A seleção dos sensores que serão incluídos como entrada é de grande importância em modelo não redundantes. Modelos construídos com grupos de sensores cujas medidas apresentam correlações altas geram menor erro de predição quando comparados com os modelos que apresentam uma baixa correlação nos vetores de entrada (Hines et al., 2000; Najafi et al., 2004; Kramer, 1991).

A Figura 2 mostra o diagrama de blocos do modelo de um sistema de monitoramento on-line. O vetor de medidas dos sensores $\mathbf{X}$ é a entrada para o modelo de auto-correção, que calcula a melhor estimativa do vetor de entrada. $\mathrm{O}$ vetor estimado é comparado com o vetor de entrada, criando o vetor de resíduos r, que será a entrada do modelo de lógica de decisão. Este determina se o resíduo é estatisticamente diferente de zero e se está dentro da faixa de tolerância; sua saída é uma indicação do estado de cada sensor.

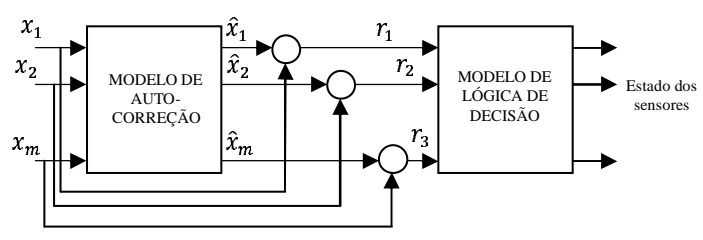

Figura 2: Modelo de Monitoramento on-line.

O modelo proposto neste trabalho visa a calcular os parâmetros estimados dos sensores empregando os dados históricos de um conjunto de sensores correlacionados.

\subsection{Modelo de Auto-Correção}

O objetivo do modelo é corrigir as medidas de um conjunto de instrumentos quando estes apresentam alguma degradação no seu estado, devido a uma instalação inadequada, ao envelhecimento do sensor ou a uma deformação do sensor originada por uma exposição em ambientes inadequados. 
O modelo de auto-correção tem como elemento principal a Rede Neural Auto-Associativa (RNAA), que realiza a reconstrução dos sinais. Conforme mencionado na seção 2, a RNAA atua como uma matriz identidade quando os sensores não apresentam falha, e como uma função não linear que reconstrói os sinais quando estes apresentem alguma degradação. Assim, o modelo desenvolvido neste trabalho é considerado do tipo não redundante, pois utiliza os dados históricos do funcionamento dos sensores e cobre toda a gama de funcionamento do processo.

O desenvolvimento do modelo de auto-correção compreende os seguintes passos.

\section{Passo 1 - Correlação dos sensores}

Nesta primeira etapa, analisa-se a correlação das medidas dos sensores. O grau de correlação entre as variáveis é um aspecto significativo para que uma RNAA trabalhe como um sistema de monitoramento (Wrest et al., 1996; Kramer, 1991). Em situações de correlação alta, desvios, offset ou ruídos em um dos sensores não afetam significativamente a resposta da RNAA, pois a saída está relacionada com todas as entradas através de um grande número de pesos e padrões. O grau de coerência entre parâmetros $i$ e $j$ é determinado pela função de correlação $S(i, j)=\frac{C(i, j)}{\sqrt{C(i, i) C(j, j)}}$ onde $C$ representa a matriz de covariância. Valores próximos a \pm 1 representam alta correlação na faixa de trabalho dos sensores.

Neste estudo, verificou-se que, apesar de os sensores realizarem o monitoramento de uma mesma planta, nem sempre todos apresentaram correlações altas. Assim, quando a quantidade de sensores é grande e as correlações não são uniformes, propõe-se realizar uma divisão do conjunto de sensores e criar grupos que apresentem correlações similares, com a finalidade de obter uma melhor aproximação dos sinais.

\section{Passo 2 - Pré-processamento de dados}

Efetuada a identificação dos subgrupos de sensores, um tratamento da base de dados deve ser realizado: retiram-se dados inconsistentes ou incompletos antes do treinamento da RNAA. Em seguida, realiza-se uma normalização dos dados. No caso deste trabalho, em que a função de ativação dos neurônios nas camadas escondidas é do tipo tansig (tangente sigmóide), as medidas foram mapeadas em uma faixa de $[-1,1]$ para simplificar o treinamento.

\section{Passo 3 - Estimativa de neurônios}

A complexidade da RNAA é definida pelo número de neurônios na camada de estrangulamento (Kramer, 1991; Hines and Garvey, 2007); embora o número de neurônios nas camadas de mapeamento e desmapeamento afete o desempenho da rede, alterações nos neurônios na camada de estrangulamento terão efeito mais significativo na qualidade da resposta. Para encontrar o número de neurônios nesta camada, treinam-se e testam-se distintas topologias com a finalidade de colher informações para avaliar o número de neurônios que forneça à RNAA a capacidade de corrigir as medidas de um sensor. A RNAA deve ser treinada como uma base de dados que apresente perturbações, conforme explicado na seção 2.1. O número de neurônios nas camadas de mapeamento e desmapeamento é então escolhido com a finalidade de se obter a resposta desejada livre de ruídos (um número maior de neurônios proporciona, em geral, uma filtragem superior). A rede terá, então, a capacidade de reconstruir os sinais de um sensor com o auxílio da correlação existente com os outros sensores.

Ao contrário de estudos anteriores, neste trabalho foi avaliada a resposta fornecida pela rede para cada sinal reconstruído. O treinamento da rede é realizado com base na média dos erros quadráticos (MSE). A decisão sobre o melhor modelo é efetuada via validação cruzada (Haykin, 1998), com base no valor médio do erro percentual (MAPE). Na fase de testes, é também utilizada, para avaliação do desempenho, a raiz quadrada do erro médio quadrático (RMSE). As expressões para estas métricas são as seguintes:

$$
\begin{aligned}
M S E & =\frac{\sum_{i=1}^{N}\left(x_{i}-\hat{x}_{i}\right)^{2}}{N} \\
R M S E & =\sqrt{\frac{\sum_{i=1}^{N}\left(x_{i}-\hat{x}_{i}\right)^{2}}{N}} \\
M A P E & =\frac{1}{N} \sum_{i=1}^{N}\left|\frac{x_{i}-\hat{x}_{i}}{x_{i}}\right| \times 100 \%
\end{aligned}
$$

\section{Passo 4 - Treinamento Robusto Modificado (RNAAM)}

Neste passo, almeja-se fornecer à rede a capacidade de reconstrução de sinais que apresentam erros súbitos ao mesmo tempo. O treinamento robusto proposto por Kramer é capaz de reconstruir os sinais dos sensores que apresentam falhas, desde que estes não sejam simultâneas, utilizando uma ou mais redes neurais auto-associativas (Marseguerra and Zoia, 2005b; Hines et al., 2000; Simani et al., 2000; Böhme et al., 1999). A RNAA robusta consegue realizar a reconstrução dos sinais dos sensores quando um único sensor do grupo apresenta falha, mas, no momento de reconstruir o sinal com falha, observam-se desvios nas saídas correspondentes aos sensores que não apresentaram falhas. 
Com o objetivo de melhorar a resposta da RNAA no caso de múltiplas falhas e de desenvolver uma metodologia passível de ser empregada em sistemas reais, propõe-se, neste trabalho, uma modificação do treinamento robusto. Considere-se um conjunto de treinamento $\mathbf{X}=\left[\mathbf{X}_{1 \times n}, \mathbf{X}_{2 \times n}, \ldots \mathbf{X}_{m \times n}\right]$, onde $\mathbf{X}_{i \times n}$ representa o vetor de $n$ amostras do sensor $i$. Inserindo-se ruído apenas nas medidas de um sensor $i$, por exemplo, observa-se, na saída da rede neural, como se dá a reconstrução dos sinais de todos os sensores. Supondo que as medidas de outros dois sensores ( $i+2 \mathrm{e} i+4$, por exemplo) não tenham sido reconstruídas satisfatoriamente, inserem-se, na entrada da rede, em uma etapa seguinte, ruídos aleatórios nos sinais destes dois sensores. Repete-se este procedimento para outros dois sensores, até que os dados de todos eles, sempre dois a dois, tenham sido apresentados de forma corrompida à rede. A rede com tal treinamento recebe o nome de RNAAM (Rede Neural Auto-Associativa Modificada).

\subsection{Modelo de Lógica de Decisão}

Tem-se como objetivo detectar falha em sensores o mais rapidamente possível, com uma probabilidade pequena de tomar uma decisão errada. O modelo usado neste trabalho para atingir tal objetivo faz uso do teste de probabilidade de relação seqüencial (SPRT). Esta metodologia detecta a região de operação do sensor com base nos tempos de falha. O algoritmo usa o resíduo como entrada para avaliar o estado do sensor. Ao contrário de testes com comprimento fixos de tempo que calculam uma nova média e variância em cada época de amostragem, o algoritmo monitora continuamente o desempenho do sensor através do processamento do resíduo. Devido à sua natureza seqüencial, necessita de um número mínimo de amostras para calcular o erro de tolerância nos procedimentos de detecção.

No SPRT, considera-se que o resíduo obtido apresenta uma distribuição de probabilidade normal com média $(\mu)$ zero e variância $\left(\sigma^{2}\right)$ igual às pequenas variações características de um sinal de campo produto de um ruído atenuado. A função de distribuição de probabilidade do resíduo é do tipo:

$$
p\left(x \mid H_{0}\right)=\frac{1}{\sqrt{2 \pi \sigma^{2}}} \exp \left(-\frac{(x-\mu)^{2}}{2 \sigma^{2}}\right)
$$

O SPRT é usado para decidir entre duas hipóteses, $H_{0}$ e $H_{1}$. $H_{1}$ corresponde às regiões de taxa decrescente da falha (TDF) ou à taxa de aumento da falha (TAF), e $H_{0}$ denota uma região de taxa de modo normal (TMN). Isto permite a detecção quando o resíduo ultrapassa a região TMN, tanto para a região TDF como para a região TAF.

Para calcular o SPRT, é necessário primeiramente computar a razão de verossimilhança, representada pela equação (3).

$$
\begin{aligned}
L_{n} & =\frac{\text { prob. de observacao }\left\{X_{n}\right\} \text { dado } H_{1} \text { verdadeira }}{\text { prob. de observacao }\left\{X_{n}\right\} \text { dado } H_{0} \text { verdadeira }} \\
& =\frac{p\left(\left\{X_{n}\right\} \mid H_{1}\right)}{p\left(\left\{X_{n}\right\} \mid H_{0}\right)}
\end{aligned}
$$

onde $\mathbf{X}_{n}$ é uma sequiência de $\mathrm{n}$ observações consecutivas de $\mathrm{X}$.

A razão de verossimilhança é, então, comparada com o limite inferior $A$ e o limite superior $B$, (cf. equação (4)) definidos pela probabilidade de alarme falso $(\alpha)$ e probabilidade de ausência de alarme $(\beta)$ :

$$
\begin{aligned}
& A=\frac{\beta}{1-\alpha} \\
& B=\frac{1-\beta}{\alpha}
\end{aligned}
$$

Se a razão de verossimilhança for menor do que $A$, ela pertence à região TNM do sistema; se for maior do que $B$, pertence à região degradada do sistema (TDF ou TAF), e uma falha é registrada (cf. Figura 3).

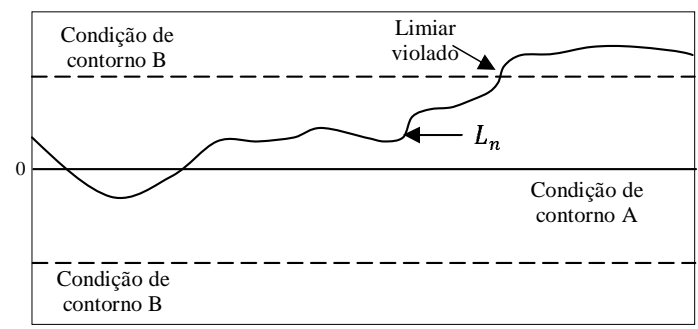

Figura 3: Comportamento do resíduo frente ao SPRT.

\section{ESTUDO DE CASO}

O estudo de caso foi escolhido com o objetivo de mostrar que a RNAAM realiza uma reconstrução on-line dos sinais dos sensores quando estes apresentam desvio, offset e ruído. Recorde-se que o modelo proposto é da categoria não redundante, efetivo para realizar monitoramento de sensores em nível industrial, e apropriado para se avaliar estados de sensores ao longo do tempo.

O modelo foi avaliado com uma base dados de medidas de sensores que controlam e realizam o monitoramento de um motor de combustão interna acoplado a um alternador, que gera a energia necessária para alimentar dois motores elétricos encarregados da tração das rodas traseiras de um caminhão de mineração da empresa Barrick, no Peru. 
A primeira etapa do trabalho foi confirmar que os sensores estavam previamente calibrados, de forma a se realizarem, então, simulações de falhas. A base de dados fornecida apresenta valores filtrados previamente na etapa de aquisição de sinal no ECM (Engine Control Module). Portanto, as medidas dos sensores que apresentam variações abruptas são consideradas parte da operação do motor e não ruídos externos.

Das 40 variáveis de medidas fornecidas, foram selecionadas 32 , correspondentes às medidas de distintos sensores de pressão e temperatura instalados exteriormente ao motor. Foram desconsideradas as variáveis produto de cálculos feitos no ECM, medições do tipo PWM (Pulse Width Modulation) e sensores com saída do tipo ON-OFF.

Os dados representam medidas relativas ao translado de produtos pelo caminhão. O sistema de monitoramento do caminhão gerou uma base de dados de 2000 amostras, com medições realizadas com um período de amostragem de 1 minuto para cada sensor. Diferentemente de outros estudos, os sinais produzidos pelos sensores não são ideais, ou seja, apresentam variações resultantes do esforço do motor durante o translado de material.

Das 2000 amostras, utilizaram-se 800 para a etapa de treinamento, 200 para validação, e 1000 para a etapa de teste. De forma a evitar o super-treinamento (overfitting), foi utilizada a técnica de parada antecipada ou prematura (earlystopping) para o treinamento de todas as redes neurais testadas.

Com base na metodologia descrita na seção 3, analisaram-se as medidas para identificar grupos de sensores com correlações similares. Os sensores foram divididos em três grupos, associando-se a cada um deles uma RNAAM. A Figura 4 apresenta um diagrama de blocos do modelo de autocorreção previsto. Detalham-se, a seguir os testes efetuados com cada um dos grupos.

\subsection{Teste com o Modelo de Auto- Correção}

\section{Grupo 1}

Os sensores selecionados para formar este grupo, apresentados na Tabela 1, medem a temperatura na câmara de combustão interna dos oito cilindros do motor e apresentaram um grau de correlação acima de 0,87 .

Na elaboração de um algoritmo para buscar o menor número de neurônios na camada de estrangulamento, testaramse distintas topologias para a RNAAM, variando-se o número de neurônios nas camadas escondidas e inserindo-se ruído nas medidas. Com base nos erros obtidos na etapa

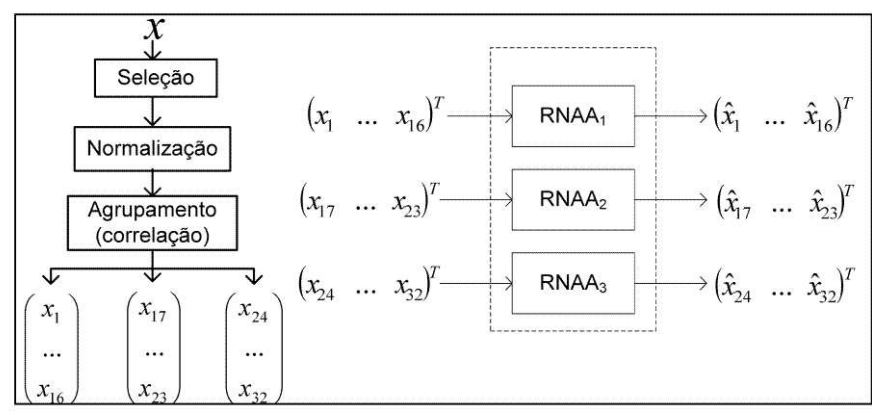

Figura 4: Modelo de Auto-Correção.

Tabela 1: Sensores de Temperatura nos Cilindros - Gr. 1.

\begin{tabular}{|l|l|}
\hline \multicolumn{2}{|c|}{ Sensores do Grupo 1 } \\
\hline T. de escape cilindro 1 LB & T. de escape cilindro 5 LB \\
\hline T. de escape cilindro 1 RB & T. de escape cilindro 5 RB \\
\hline T. de escape cilindro 2 LB & T. de escape cilindro 6 LB \\
\hline T. de escape cilindro 2 RB & T. de escape cilindro 6 RB \\
\hline T. de escape cilindro 3 LB & T. de escape cilindro 7 LB \\
\hline T. de escape cilindro 3 RB & T. de escape cilindro 7 RB \\
\hline T. de escape cilindro 4 LB & T. de escape cilindro 8 LB \\
\hline T. de escape cilindro 4 RB & T. de escape cilindro 8 RB \\
\hline
\end{tabular}

de validação, a rede com maior capacidade de generalização foi escolhida via validação cruzada. Ao final do treinamento, foi testada a capacidade da rede de se comportar como um filtro - ou corretor de sinais - frente às medidas com ruído. A Figura 5 resume os resultados alcançados, na fase de treinamento, para as diferentes topologias escolhidas e mostra o comportamento da RNAAM em função do número de neurônios na camada de estrangulamento (de 1 a 14), considerando-se diferentes números de neurônios nas camadas de mapeamento e desmapeamento (identificados por M no quadro superior direito).

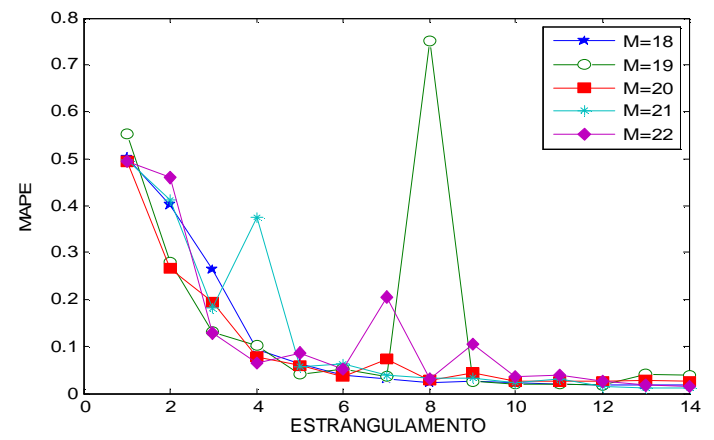

Figura 5: Erro (MAPE) em função do número de neurônios na camada de estrangulamento. 
Observa-se que, independentemente do número de neurônios nas camadas de mapeamento e desmapeamento, a rede gera menor erro na predição quando o número de neurônios na camada de estrangulamento atinge um valor próximo do número de entradas. Embora um maior número de neurônios na camada de estrangulamento melhore a capacidade da rede de operar frente a perturbações em um sensor, o custo computacional para calcular os pesos da rede é elevado. Escolher o menor número de neurônios nesta camada que possa fornecer uma boa aproximação da saída (sem perturbação) é imprescindível para reduzir o custo computacional e obter uma boa generalização. Na maioria das topologias testadas, quando a camada de estrangulamento supera os seis neurônios, o erro de predição permanece praticamente o mesmo, ou seja, a variação é insignificante. Desta forma, foram escolhidos 6 neurônios para esta camada.

O passo seguinte foi treinar a RNAAM para reconstrução de medidas quando estas apresentam ruídos, desvios ou offset nos vetores de entrada. Para tal, foi usado o treinamento robusto modificado (seção 3.1) com ruído aleatório de $10 \%$ da faixa de medição. O número de neurônios nas camadas de mapeamento e desmapeamento foi aumentado até se obter uma resposta com erro MAPE máximo de $2 \%$. Ao final do treinamento, a topologia obtida apresentou 18 neurônios na camada de mapeamento/desmapeamento. A Tabela 2 mostra os resultados obtidos com a topologia 16-18-6-18-16. Observa-se que o MAPE é menor do que $1 \%$ para todos os sensores, atingindo-se, assim, o objetivo proposto.

A rede foi testada quanto à reconstrução de sinais frente a erros súbitos. Foram apresentados vetores de entrada com um desvio de $0,7 \%$ por minuto $\left(5^{\circ} \mathrm{F}\right)$ durante 500 minutos (ao final do processo, o desvio é de $100^{\circ} \mathrm{F}$ ). A Figura 6 apresenta o resultado da RNAAM 1 frente ao desvio do sensor 1 e o comportamento para o sensor 2 do mesmo grupo; a Tabela 3 apresenta os resultados para os 16 sensores.

Os resultados mostram que o sensor 1 (Temperatura de escape cilindro 1LB) apresenta um aumento do MAPE de $0,03 \%$ para aproximadamente $0,6 \%$, abaixo do limite do erro estabelecido para o conjunto de sensores (2\%). Além disso, a média da diferença existente entre a resposta da RNAAM , $_{1}$ para os 15 sensores restantes, quando as medidas não apresentam perturbações e quando existe um desvio no sensor 1 , é de $0,21 \%$ para $0,24 \%$, ou seja, a rede não gera alterações consideráveis nas respostas dos sensores que não apresentaram falhas na entrada.

Foi testada, a seguir, a capacidade da rede de corrigir falhas em três ou mais sensores defeituosos. Dados correspondentes a medições de três sensores com falhas, selecionados aleatoriamente, foram apresentados à $\mathrm{RNAAM}_{1}$ (com desvio idêntico ao anterior). A Tabela 4 resume os resultados para
Tabela 2: Resultados sem perturbações.

\begin{tabular}{|c|c|c|c|}
\hline Grupo I & \multicolumn{3}{|c|}{ 16-18-6-18-16 } \\
\hline Sensor & MSE (e-3) & MAPE (\%) & RMSE \\
\hline 1 & 0,1500 & 0,0392 & 0,7618 \\
\hline 2 & 0,1030 & 0,1666 & 2,5842 \\
\hline 3 & 0,2010 & 0,2244 & 3,0304 \\
\hline 4 & 0,1100 & 0,1522 & 2,2418 \\
\hline 5 & 0,1400 & 0,1826 & 2,6020 \\
\hline 6 & 0,0200 & 0,0518 & 1,0336 \\
\hline 7 & 0,1920 & 0,1936 & 2,6275 \\
\hline 8 & 0,1700 & 0,2023 & 2,8081 \\
\hline 9 & 0,3400 & 0,3184 & 4,3957 \\
\hline 10 & 0,2010 & 0,2378 & 3,3871 \\
\hline 11 & 0,2180 & 0,2426 & 3,6817 \\
\hline 12 & 0,1520 & 0,2030 & 2,9606 \\
\hline 13 & 0,2800 & 0,2512 & 3,6144 \\
\hline 14 & 0,1890 & 0,2463 & 3,6886 \\
\hline 15 & 0,2660 & 0,2495 & 3,4549 \\
\hline 16 & 0,1950 & 0,2416 & 3,4527 \\
\hline
\end{tabular}

Tabela 3: Desvio de 0,7\% por minuto no sensor 1.

\begin{tabular}{|c|c|c|c|}
\hline Grupo I & \multicolumn{3}{|c|}{ 16-18-6-18-16 } \\
\hline Sensor & MSE (e-3) & MAPE (\%) & RMSE \\
\hline $\mathbf{1}$ & $\mathbf{0 , 0 0 1 7}$ & $\mathbf{0 , 6 2 3 3}$ & $\mathbf{8 , 0 3 0 8}$ \\
\hline 2 & 0,0002 & 0,2131 & 3,2284 \\
\hline 3 & 0,0002 & 0,2208 & 3,0248 \\
\hline 4 & 0,0001 & 0,153 & 2,2289 \\
\hline 5 & 0,0003 & 0,2775 & 3,7958 \\
\hline 6 & 0 & 0,0466 & 0,8067 \\
\hline 7 & 0,0002 & 0,1864 & 2,5327 \\
\hline 8 & 0,0002 & 0,2093 & 2,8926 \\
\hline 9 & 0,0003 & 0,3172 & 4,315 \\
\hline 10 & 0,0005 & 0,3882 & 5,3334 \\
\hline 11 & 0,0002 & 0,2481 & 3,7299 \\
\hline 12 & 0,0004 & 0,337 & 4,5454 \\
\hline 13 & 0,0002 & 0,2357 & 3,3597 \\
\hline 14 & 0,0002 & 0,2335 & 3,4417 \\
\hline 15 & 0,0005 & 0,3425 & 4,5410 \\
\hline 16 & 0,0002 & 0,2586 & 3,8134 \\
\hline
\end{tabular}


todos os sensores, considerando-se os sensores 1 (Temperatura de escape cilindro $1 \mathrm{LB}), 5$ (Temperatura de escape cilindro 3 LB) e 11 (Temperatura de escape cilindro 6 LB) como aqueles com falhas. A Figura 7 apresenta a resposta da RNAAM $_{1}$ para os sensores 1 e 2 frente aos desvios nos sensores 1,5 e 11 . Pode-se perceber que a $\mathrm{RNAAM}_{1}$ consegue efetuar a auto-correção das medidas dos sensores que apresentaram desvios com um valor de MAPE inferior a $1 \%$. Contrastando-se os valores das Tabelas 3 e 4, observa-se que a máxima diferença entre os dois casos é apresentada pelo sensor 5 - aumento do MAPE de $0,277 \%$ para $0,729 \%$.

Finalmente, testou-se a RNAAM $_{1}$ com ruído aleatório de $20 \%$ da faixa de medição, que pode ser considerado como causado por um evento externo que interfere no hardware do sensor. Como exemplo, foi inserido nos sinais do sensor 1 (Temperatura de escape Cilindro 1LB) um ruído aleatório gaussiano com média 0 e desvio padrão equivalente a $40^{\circ} \mathrm{F}$. A Figura 8 mostra que a RNAAM 1 consegue filtrar o ruído satisfatoriamente.

Tabela 4: Desvio em 100 unidades dos sensores 1, 5 e 11.

\begin{tabular}{|c|c|c|c|}
\hline Grupo I & \multicolumn{3}{|c|}{ 16-18-6-18-16 } \\
\hline Sensor & MSE (e-3) & MAPE (\%) & RMSE \\
\hline $\mathbf{1}$ & $\mathbf{0 , 0 0 1 9}$ & $\mathbf{0 , 6 5 5 0}$ & $\mathbf{6 , 4 4 3 6}$ \\
\hline 2 & 0,0002 & 0,2550 & 3,7725 \\
\hline 3 & 0,0002 & 0,2366 & 3,2450 \\
\hline 4 & 0,0004 & 0,2622 & 4,0100 \\
\hline $\mathbf{5}$ & $\mathbf{0 , 0 0 2 1}$ & $\mathbf{0 , 7 2 9 9}$ & $\mathbf{7 , 9 1 7 4}$ \\
\hline 6 & 0 & 0,0880 & 1,4911 \\
\hline 7 & 0,0006 & 0,3436 & 4,4874 \\
\hline 8 & 0,0003 & 0,2727 & 3,7162 \\
\hline 9 & 0,0005 & 0,3862 & 5,2263 \\
10 & 0,0008 & 0,4989 & 6,6872 \\
\hline $\mathbf{1 1}$ & $\mathbf{0 , 0 0 0 3}$ & $\mathbf{0 , 2 9 9 1}$ & $\mathbf{4 , 5 1 2 1}$ \\
\hline 12 & 0,0006 & 0,4446 & 6,0273 \\
\hline 13 & 0,0003 & 0,2389 & 3,6136 \\
\hline 14 & 0,0004 & 0,4016 & 5,6880 \\
\hline 15 & 0,001 & 0,5091 & 6,5595 \\
\hline 16 & 0,0006 & 0,4262 & 6,1295 \\
\hline
\end{tabular}

\section{Grupo 2}

Os sensores do Grupo 2 (Tabela 5) apresentaram um grau de correlação de aproximadamente 0,6 .

Seguindo a metodologia proposta na seção 3, foram testadas, primeiramente, distintas topologias para se obter o melhor
Tabela 5: Sensores de Temperatura e Pressão - Grupo 2.

\begin{tabular}{|c|l|}
\hline $\mathrm{N}^{\circ}$ & \multicolumn{1}{|c|}{ Sensores Grupo 2} \\
\hline 1 & P. do ar na saída do turbo de alta esquerdo \\
\hline 2 & P. do ar na saída do turbo de alta direito \\
\hline 3 & $\begin{array}{l}\text { Temperatura do ar no aftercooler } \text { de frente } \\
\text { esquerdo }\end{array}$ \\
\hline 4 & $\begin{array}{l}\text { Temperatura do ar no aftercooler } \text { posterior } \\
\text { esquerdo }\end{array}$ \\
\hline 5 & Temperatura do ar no aftercooler de frente direito \\
\hline 6 & Temperatura do ar no aftercooler posterior direito \\
\hline 7 & Pressão do refrigerante motor \\
\hline
\end{tabular}

número de neurônios na camada de estrangulamento, sempre com o objetivo de filtragem do ruído e de auto- correção das medidas de um sensor com falha. Foram considerados de 9 a 16 neurônios para as camadas de mapeamento e desmapeamento, e de 1 a 6 neurônios na camada de estrangulamento. Recorde-se que o número de neurônios nesta camada é escolhido tendo-se em vista o custo computacional, a variação do erro frente ao aumento do número de neurônios e a capacidade da rede de corrigir as entradas quando estas apresentam erros. A seguir, realizou-se o treinamento modificado, mediante a inserção, nos vetores de entradas, de erros equivalentes a $20 \%$ da faixa de medição dos sensores. O número de neurônios nas camadas de mapeamento e desmapeamento foi aumentado até se obter uma resposta com erro MAPE máximo de $2 \%$. Ao final do aprendizado, a topologia obtida apresentou 15 neurônios na camada de mapeamento e desmapeamento e 3 neurônios na camada bottleneck. A Tabela 6 mostra os resultados obtidos na topologia 7-15-3-15-7 da RNAAM $_{2}$ para os 7 sensores. Conforme se observa, o erro MAPE é menor do que $1 \%$ para todos os sensores.

Tabela 6: Resposta da RNAA do Grupo 2.

\begin{tabular}{|c|c|c|c|}
\hline Grupo II & \multicolumn{3}{|c|}{ 7-15-3-15-7 } \\
\hline Sensor & MSE & MAPE (\%) & RMSE \\
\hline 1 & 0,0060 & 0,5881 & 0,3075 \\
\hline 2 & 0,0056 & 0,5901 & 0,2907 \\
\hline 3 & 0,0019 & 0,4154 & 0,8958 \\
\hline 4 & 0,0077 & 0,3233 & 0,6789 \\
\hline 5 & 0,0037 & 0,6845 & 1,4887 \\
\hline 6 & 0,0033 & 0,4406 & 0,8563 \\
\hline 7 & 0,0087 & 0,5917 & 0,3823 \\
\hline
\end{tabular}

O passo seguinte foi testar a capacidade da rede de reconstruir os sinais frente a um sensor com falha. Para tal, foram 

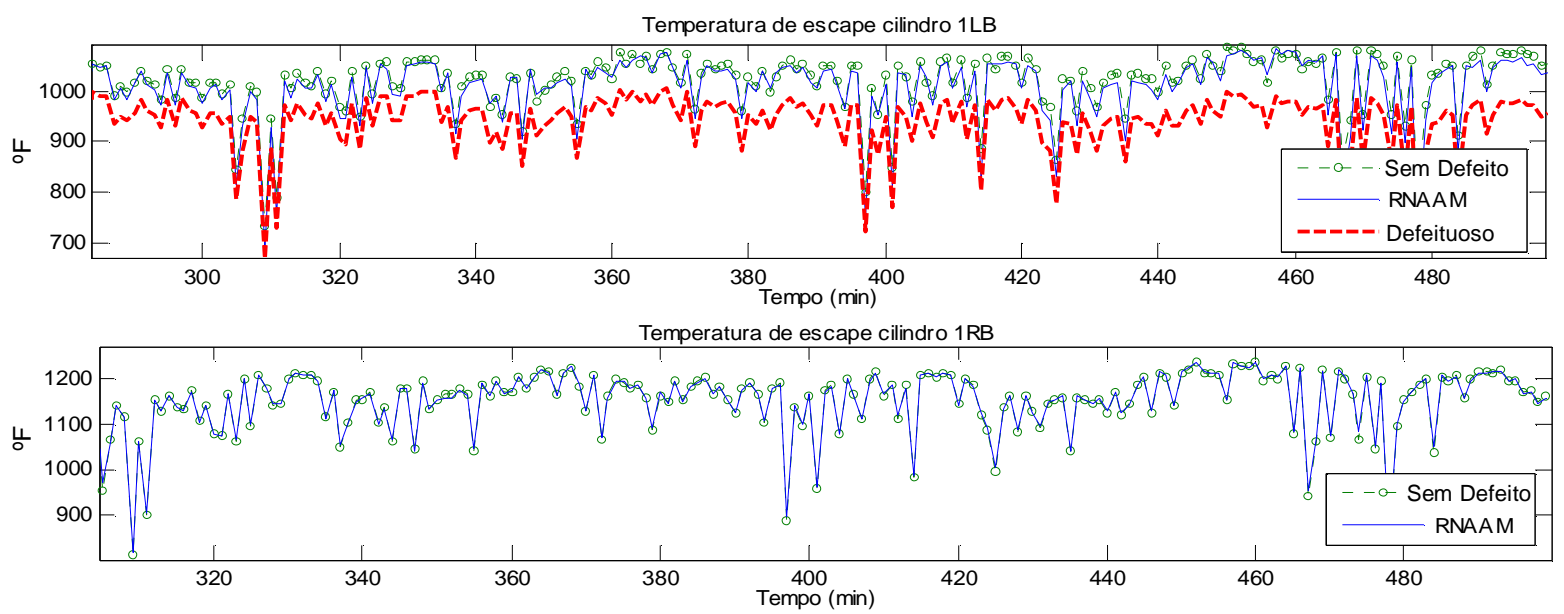

Figura 6: Resposta dos sensores 1 e 2 frente ao desvio total de $100^{\circ} \mathrm{F}$ no sensor 1 (Temperatura de escape cilindro $1 \mathrm{LB}$ ).

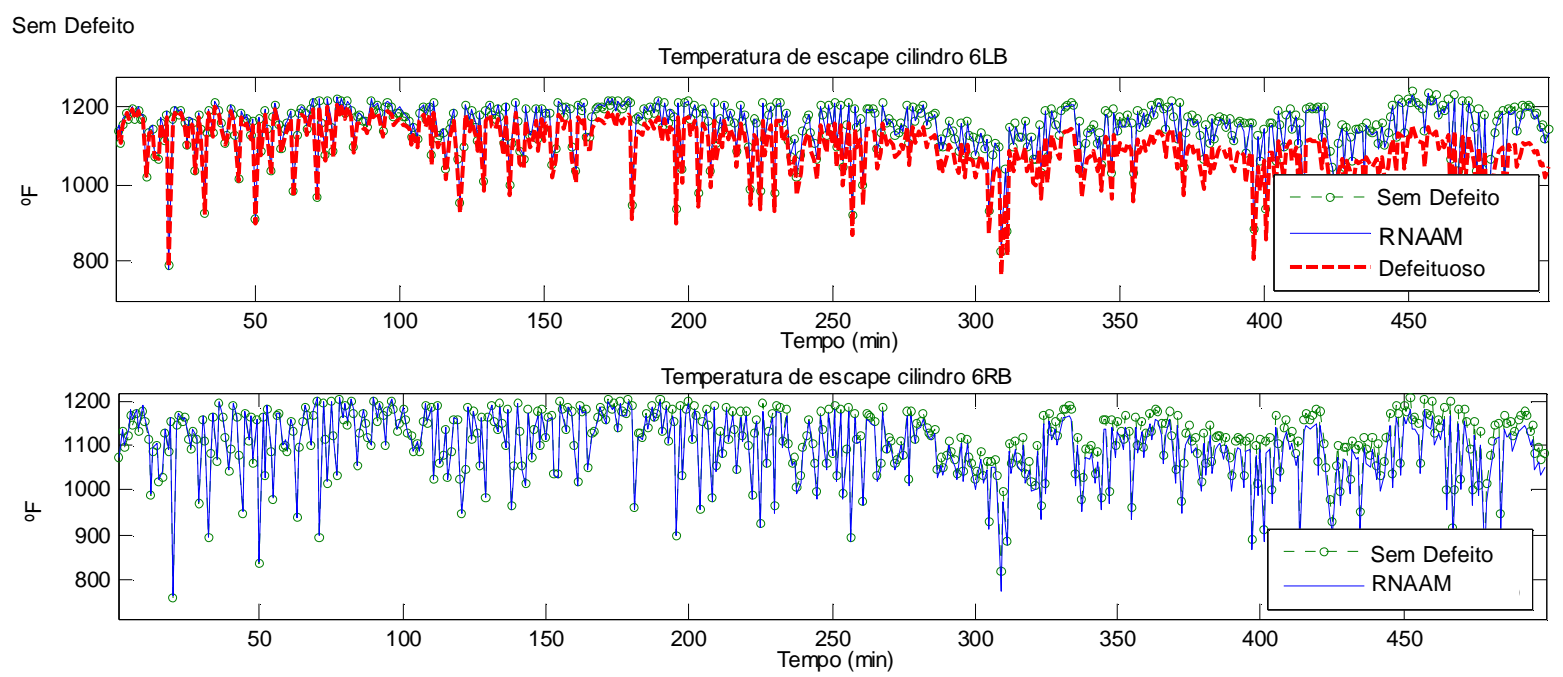

Figura 7: Resposta dos sensores frente ao desvio lento de $0,7 \%$ por minuto nos sensores 1,5 e 11 .

apresentados à rede erros do tipo offset nos sinais dos sensores. A Figura 9 apresenta as saídas da $\mathrm{RNAAM}_{2}$ para os sensores 1 (Pressão da saída do turbo de alta esquerdo) e 2 (Pressão da saída do turbo de alta direito), considerando- se um offset de 4 psi no sensor 2. A Tabela 7 resume os resultados.

Finalmente, a RNAAM 2 foi testada para múltiplas falhas do tipo offset. As medidas dos sensores 2, 4, 5, 6 e 7 (escolhidos aleatoriamente) foram corrompidas com um offset de 4 unidades ao mesmo tempo. A Tabela 8 resume os resultados obtidos. Comparando-se os resultados obtidos nos testes para falhas em um único sensor e para falhas múltiplas, verifica-se que a RNAAM ${ }_{2}$ consegue reconstruir os sinais dos sensores com um MAPE menor do que $2 \%$. Além disso, a média da diferença entre este caso e aquele isento de perturbações nos sinais sensores apresenta um aumento de $0,51 \%$ para $0,85 \%$ (MAPE). Portanto, os erros estão dentro do limite estabelecido para o conjunto de sensores analisado.

\section{Grupo 3}

Os sensores deste grupo, apresentados na Tabela 9, têm correlações com média de 0,33 ; como existem sensores com correlações inferiores a 0,2 , este grupo se constitui em um forte teste no que diz respeito à reconstrução dos sinais frente a erros súbitos nos sensores. A mesma metodologia foi novamente utilizada no treinamento da rede RNAAM $_{3}$. Neste caso, devido à baixa correlação existente entre os sensores, $\mathrm{o}$ número de amostras fornecidas à rede foi aumentado e, em consequência, o número de treinamentos. 


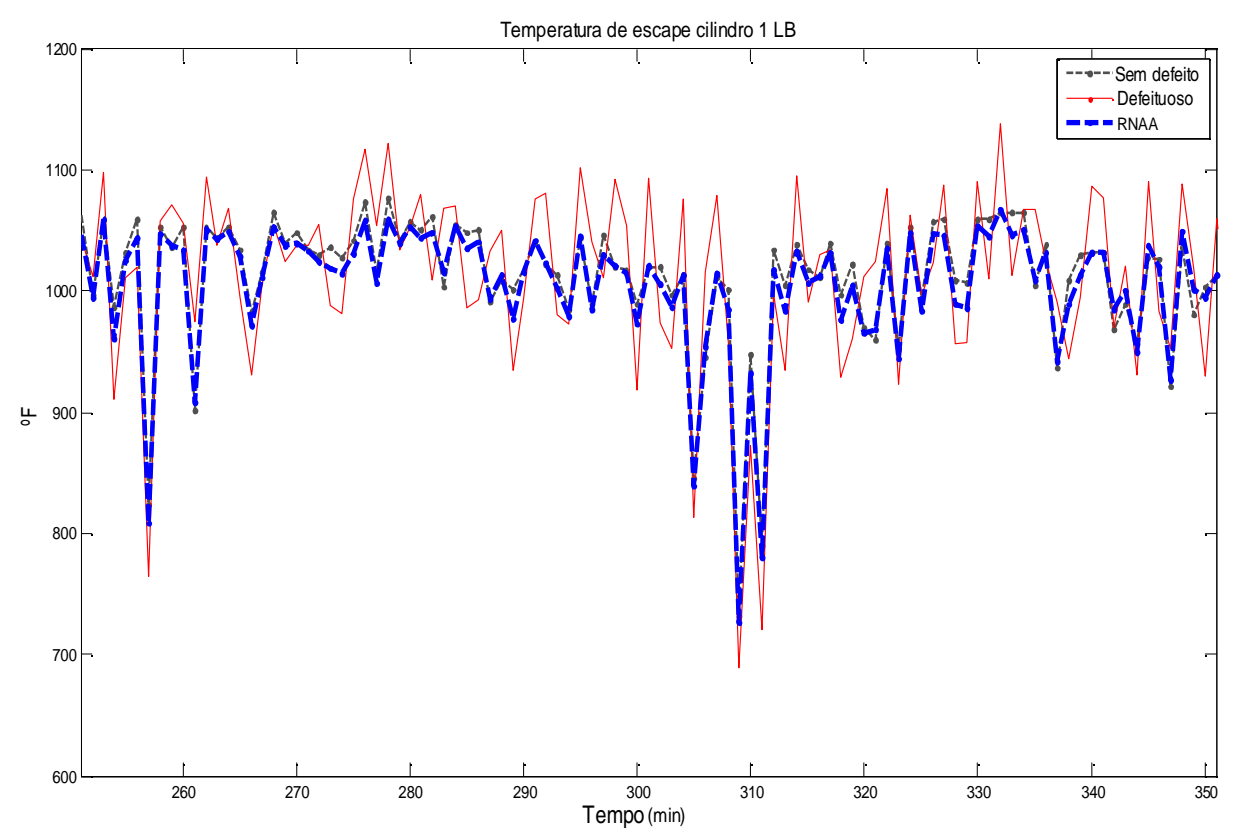

Figura 8: Resposta da $\mathrm{RNAA}_{1}$ frente ao ruído no sensor 1.

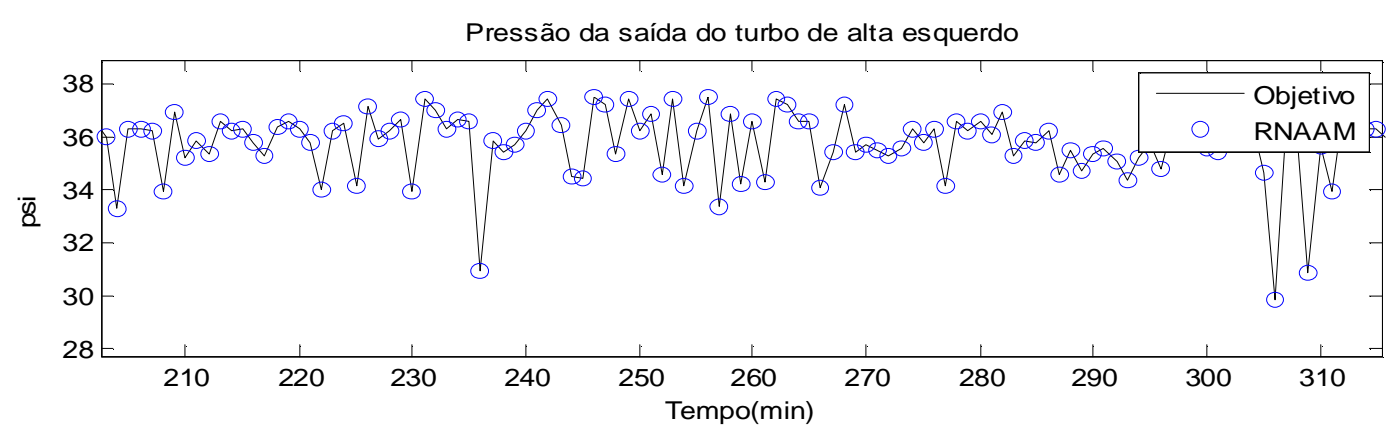

Pressão da saída do turbo de alta direito

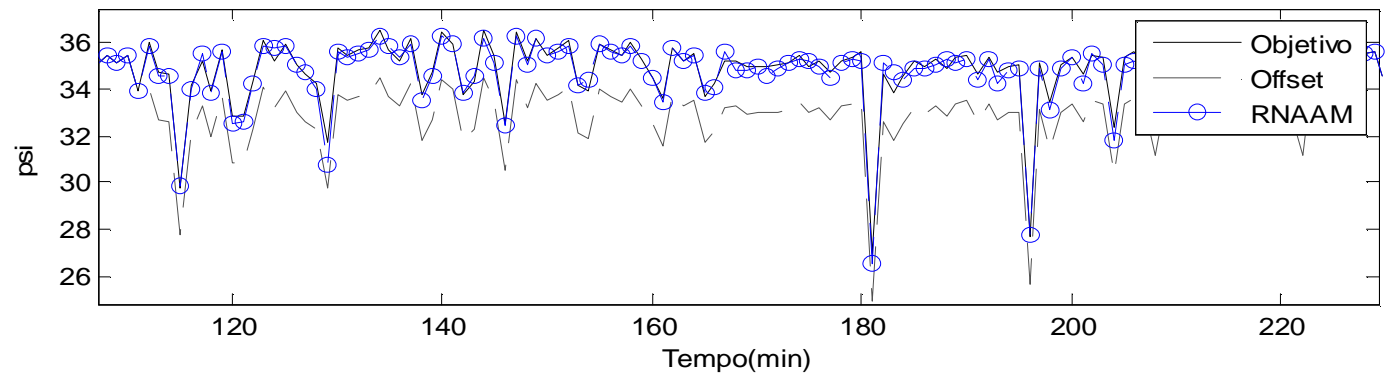

Figura 9: Respostas dos sensores 1 e 2 da $\mathrm{RNAAM}_{2}$ com falha no Sensor 2 (Offset = 4 psi).

A RNAAM 3 apresenta uma topologia 9-15-7-15-9 e a Tabela 10, a seguir, resume os resultados alcançados. Ressaltese que, devido à baixa correlação nas medidas dos sensores, $\mathrm{o}$ número de neurônios na camada de estrangulamento é maior do que nos grupos 1 e 2 , quando comparado ao número de entradas da rede.
A RNAAM ${ }_{3}$ foi testada para um erro do tipo offset de 4 psi no sensor 1 (Pressão no combustível ferroviário) nos primeiros 125 min. de operação. A Figura 10 apresenta as respostas do sensores 1 frente à falha, e a Tabela 11 resume os resultados. A resposta é satisfatória por apresentar um erro MAPE menor do que $0,02 \%$; o erro é corrigido sem alterar os demais sinais. 
Tabela 7: Resposta da RNAAM 2 a Falha do Sensor 2 (Offset $=4$ unidades).

\begin{tabular}{|c|c|c|c|}
\hline Grupo II & \multicolumn{3}{|c|}{$\mathbf{7 - 1 5 - 3 - 1 5 - 7}$} \\
\hline Sensor & MSE & MAPE (\%) & RMSE \\
\hline 1 & 0,0077 & 0,924 & 0,4278 \\
\hline $\mathbf{2}$ & $\mathbf{0 , 0 0 3 5}$ & $\mathbf{0 , 6 0 2 0}$ & $\mathbf{0 , 2 8 4 0}$ \\
\hline 3 & 0,0084 & 0,8603 & 1,6134 \\
\hline 4 & 0,0127 & 0,3595 & 0,7217 \\
\hline 5 & 0,0036 & 0,5663 & 1,2685 \\
\hline 6 & 0,0058 & 0,4390 & 0,9110 \\
\hline 7 & 0,0048 & 0,4726 & 0,2361 \\
\hline
\end{tabular}

Tabela 8: Resposta da RNAAM 2 para falhas nos sensores 2 , 4, 5, 6 e 7 (Offset $=4$ unidades).

\begin{tabular}{|c|c|c|c|}
\hline Grupo II & \multicolumn{3}{|c|}{$\mathbf{7 - 1 5 - 3 - 1 5 - 7}$} \\
\hline Sensor & MSE & MAPE (\%) & RMSE \\
\hline 1 & 0,0089 & 1,7353 & 0,7615 \\
\hline 2 & $\mathbf{0 , 0 0 5}$ & $\mathbf{1 , 2 7 2 6}$ & $\mathbf{0 , 5 6 5 1}$ \\
\hline 3 & 0,0015 & 0,3847 & 0,8300 \\
\hline 4 & $\mathbf{0 , 0 1 0 0}$ & $\mathbf{0 , 4 6 2 8}$ & $\mathbf{0 , 8 5 0 0}$ \\
\hline $\mathbf{5}$ & $\mathbf{0 , 0 0 4 4}$ & $\mathbf{0 , 8 2 0 8}$ & $\mathbf{1 , 6 9 4 0}$ \\
\hline $\mathbf{6}$ & $\mathbf{0 , 0 0 6 5}$ & $\mathbf{0 , 6 7 0 4}$ & $\mathbf{1 , 2 7 0 8}$ \\
\hline 7 & $\mathbf{0 , 0 0 4}$ & $\mathbf{0 , 6 4 0 8}$ & $\mathbf{0 , 2 4 0 5}$ \\
\hline \multicolumn{4}{|c}{} \\
\hline
\end{tabular}

Tabela 9: Sensores de Temperatura e Pressão - Grupo 2.

\begin{tabular}{|c|l|}
\hline $\mathbf{N}^{\circ}$ & \multicolumn{1}{|c|}{ Sensores Grupo 3 } \\
\hline 1 & Pressão no combustível ferroviário \\
\hline 2 & Temperatura do óleo \\
\hline 3 & Pressão do óleo na entrada do filtro \\
\hline 4 & Pressão do óleo na saída do filtro \\
\hline 5 & P. diferencial devido aos filtros de óleo \\
\hline 6 & Pressão de gases no cárter \\
\hline 7 & Temperatura do refrigerante motor \\
\hline 8 & Temperatura da entrada de ar do compressor \\
\hline 9 & Temperatura do ECM do motor \\
\hline
\end{tabular}

A rede foi testada, a seguir, para um desvio lento de $0,125 \%$ $\left(0,2^{\circ} \mathrm{F}\right)$ por minuto na medida do sensor 9 a partir do instante de tempo 500 (ao final do processo, o desvio é de $10^{\circ} \mathrm{F}$ ). A Figura 11(a) apresenta os resultados deste desvio e a Fi-
Tabela 10: Resultados da RNAAM 3.

\begin{tabular}{|c|c|c|c|}
\hline Grupo III & \multicolumn{3}{|c|}{ 9-15-7-15-9 } \\
\hline Sensor & MSE (e-6) & MAPE (\%) & RMSE \\
\hline 1 & 0,2190 & 0,0165 & 0,0329 \\
\hline 2 & 0,2078 & 0,0745 & 0,0068 \\
\hline 3 & 0,1389 & 0,2133 & 0,1666 \\
\hline 4 & 0,1234 & 0,2492 & 0,1816 \\
\hline 5 & 0,2568 & 0,0748 & 0,0046 \\
\hline 6 & 0,0506 & 0,0425 & 0,0937 \\
\hline 7 & 0,3123 & 0,0378 & 0,0840 \\
\hline 8 & 0,2808 & 0,4182 & 0,2338 \\
\hline 9 & 0,1456 & 0,0343 & 0,0560 \\
\hline
\end{tabular}

Tabela 11: Resposta da RNAAM 3 para falha no sensor 1.

\begin{tabular}{|c|c|c|c|}
\hline Grupo III & \multicolumn{3}{|c|}{ 9-15-7-15-9 } \\
\hline Sensor & MSE & MAPE (\%) & RMSE \\
\hline $\mathbf{1}$ & $\mathbf{0 , 0 0 0 0}$ & $\mathbf{0 , 0 1 2 2}$ & $\mathbf{0 , 0 3 5 7}$ \\
\hline 2 & 0,0000 & 0,0578 & 0,0055 \\
\hline 3 & 0,0001 & 0,1696 & 0,1647 \\
\hline 4 & 0,0002 & 0,2210 & 0,2087 \\
\hline 5 & 0,0000 & 0,0537 & 0,0039 \\
\hline 6 & 0,0000 & 0,0582 & 0,1489 \\
\hline 7 & 0,0000 & 0,0285 & 0,0699 \\
\hline 8 & 0,0003 & 0,2501 & 0,2011 \\
\hline 9 & 0,0000 & 0,0707 & 0,1410 \\
\hline
\end{tabular}

gura 11(b) mostra uma visão ampliada deste teste. Na Tabela 12 são resumidos os resultados para os 9 sensores da RNAAM $_{3}$.

Finalmente, a $\mathrm{RNAAM}_{3}$ foi testada para múltiplas falhas: as medidas de vários sensores foram corrompidas com um offset de 5 unidades ao mesmo tempo. A Figura 12 apresenta as respostas da RNAAM 3 para falhas simultâneas nos sensores 1, 3, 7 e 9, e na Tabela 13 são resumidos os resultados para estas falhas.

Comparando-se os resultados dos testes sem perturbações e para falhas múltiplas, verifica-se que a RNAAM ${ }_{3}$ consegue reconstruir os sinais dos sensores com um MAPE menor do que $2 \%$. Além disso, a média da diferença entre esses casos e quando não existem perturbações nos sensores é de $0,34 \%$. Portanto, os erros estão dentro do limite estabelecido para o conjunto de sensores analisado. Uma conclusão fundamental, e que atesta a validade da proposta deste trabalho, é que 


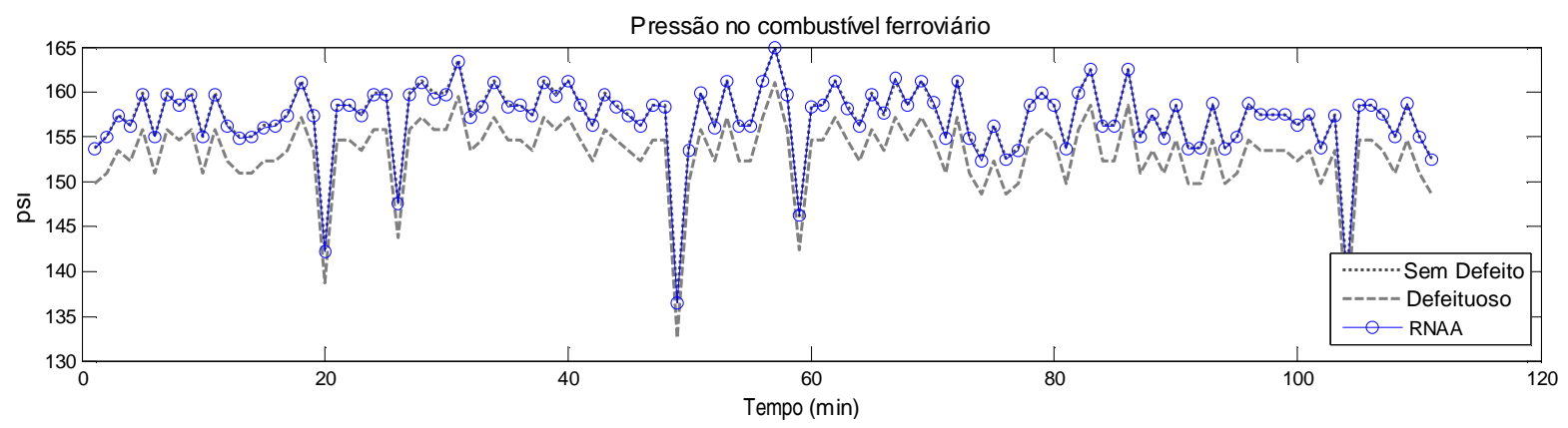

Figura 10: Respostas da RNAA frente a uma falha no sensor 1 (Offset $=4 \mathrm{psi}$ ).

Tabela 12: Resposta da RNAA 3 para o desvio no sensor 9.

\begin{tabular}{|c|c|c|c|}
\hline Grupo III & \multicolumn{3}{|c|}{ 9-15-7-15-9 } \\
\hline Sensor & MSE & MAPE (\%) & RMSE \\
\hline 1 & 0 & 0,0119 & 0,0223 \\
\hline 2 & 0 & 0,0408 & 0,0040 \\
\hline 3 & 0,0001 & 0,2010 & 0,1591 \\
\hline 4 & 0,0001 & 0,2364 & 0,1780 \\
\hline 5 & 0 & 0,0338 & 0,0020 \\
\hline 6 & 0 & 0,0382 & 0,0910 \\
\hline 7 & 0 & 0,0202 & 0,0452 \\
\hline 8 & 0 & 0,1382 & 0,0884 \\
\hline $\mathbf{9}$ & $\mathbf{0 , 0 0 1 8}$ & $\mathbf{0 , 9 2 9 1}$ & $\mathbf{1 , 5 7 5 2}$ \\
\hline
\end{tabular}

Tabela 13: Resposta da RNAAM 3 a falhas nos sensores 1, 3, 7 e 9 (offset $=5$ unidades).

\begin{tabular}{|c|c|c|c|}
\hline Grupo III & \multicolumn{3}{|c|}{ 9-15-7-15-9 } \\
\hline Sensor & MSE & MAPE (\%) & RMSE \\
\hline $\boldsymbol{1}$ & $\mathbf{0 , 0 0 6 3}$ & $\mathbf{0 , 0 1 1 8}$ & $\mathbf{0 , 0 3 5 7}$ \\
\hline 2 & 0,0058 & 0,0520 & 0,0055 \\
\hline $\mathbf{3}$ & $\mathbf{0 , 1 2 6 7}$ & $\mathbf{0 , 1 5 9 6}$ & $\mathbf{0 , 1 6 4 7}$ \\
\hline 4 & 0,2235 & 0,2087 & 0,2087 \\
\hline 5 & 0,0160 & 0,0504 & 0,0039 \\
\hline 6 & 0,0188 & 0,0553 & 0,1489 \\
\hline $\mathbf{7}$ & $\mathbf{0 , 0 2 1 7}$ & $\mathbf{0 , 0 2 7 7}$ & $\mathbf{0 , 0 6 9 9}$ \\
\hline 8 & 0,2588 & 0,2309 & 0,2011 \\
\hline $\mathbf{9}$ & $\mathbf{0 , 0 1 6 5}$ & $\mathbf{0 , 0 6 5 0}$ & $\mathbf{0 , 1 4 1}$ \\
\hline
\end{tabular}

as três redes apresentaram excelente capacidade de generalização. Quando treinadas com perturbações em medidas de apenas dois sensores, por exemplo, foram capazes de recons-

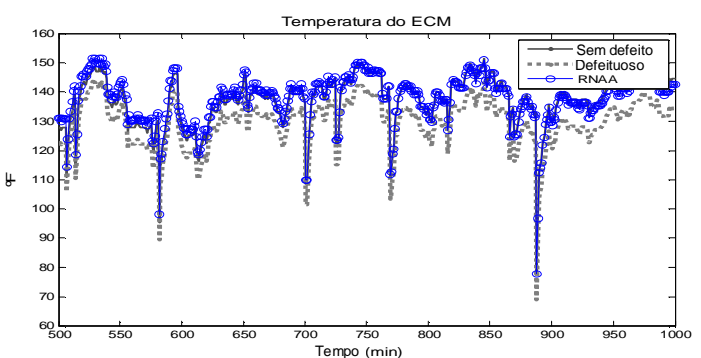

(a)

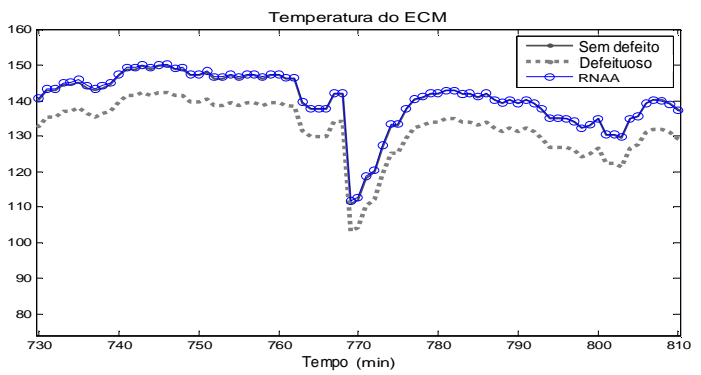

(b)

Figura 11: Respostas da RNAAM 3 frente a uma falha de desvio no sensor 9 (Desvio Final $=10^{\circ} \mathrm{F}$ ).

truir, na fase de testes, os sinais de todos os sensores, com erro mínimo.

\subsection{Resultados do Modelo de Lógica de Decisão (MLD)}

De acordo com a seção 3.2, quando um sensor está operando corretamente, o resíduo deve ter uma média aproximada de zero e uma variância comparável à dos resíduos para os dados de treinamento. Se houver um desvio nos sinais do sensor, a média residual apresentará uma mudança e, assim, a razão de verossimilhança aumentará. Se este aumento for superior ao limite definido pelo usuário, os resíduos serão mais propensos a pertencer ao modo de falha do que ao modo nor- 

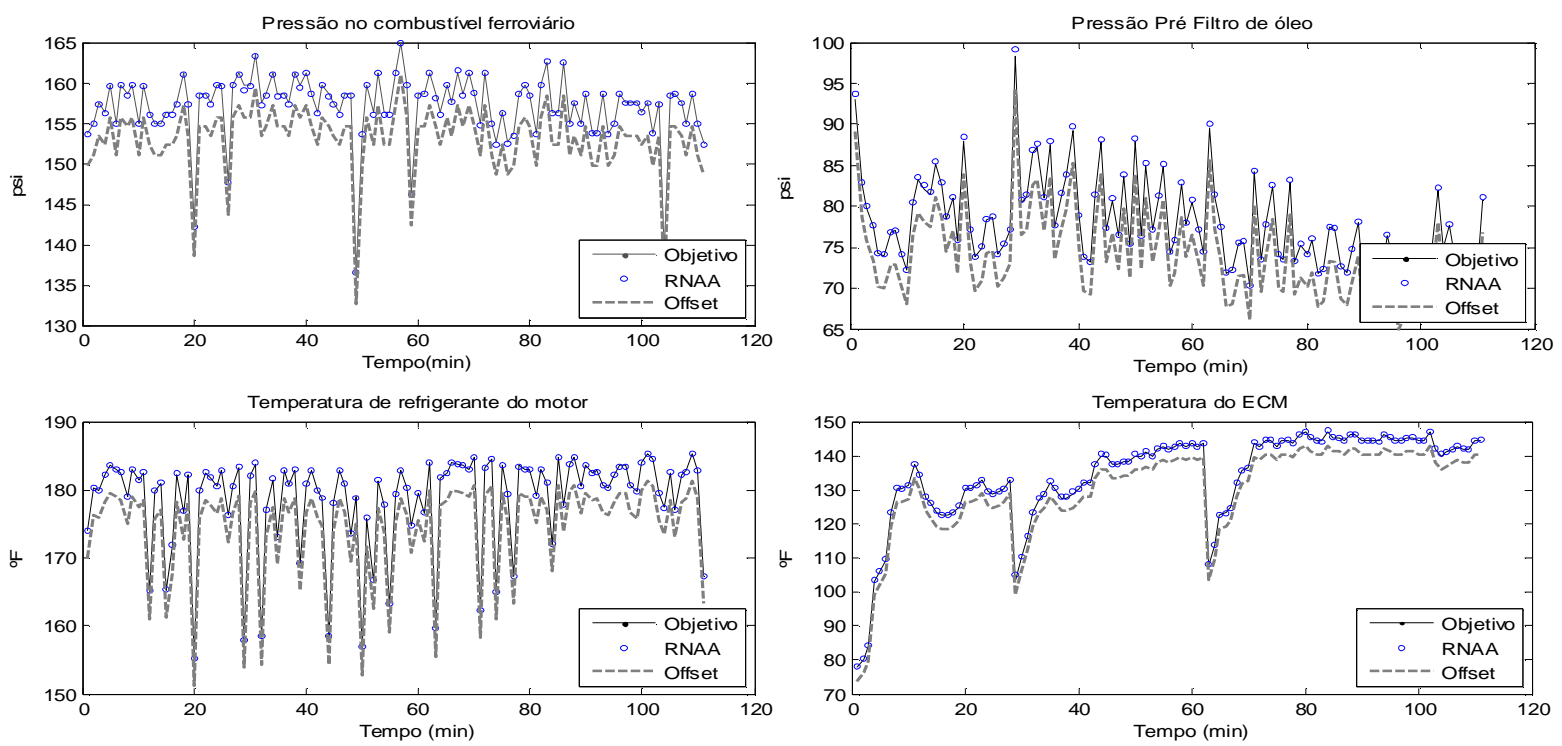

Figura 12: Resposta da $\mathrm{RNAAM}_{3}$ a falhas nos sensores 1, 3, 7 e 9 (offset $=5$ unidades).

mal (sem falha); o sensor é, então, classificado como com falha. A probabilidade de falso e a de ausência de alarme devem ser definidas pelo especialista. Assim, os limites A e $B$ da equação 6 devem ser escolhidos em função da faixa de trabalho e do tipo de sensor (Hines and Garvey, 2007).

Para testar a metodologia, foram consideradas perturbações nas medidas dos Grupos 1 e 3. Os resíduos entre as respostas geradas pelo modelo de auto-correção e as entradas foram inseridos no MLD. No primeiro teste considerou-se um erro do tipo offset de $2^{\circ} \mathrm{F}$ na medida do sensor 9/Grupo 3 (Temperatura do ECM), no minuto 500. A probabilidade de falso alarme foi fixada em 0,01 (ou 1\%), e a de ausência de alarme, em 0,1 (ou 10\%). Na Figura 13, o gráfico superior apresenta o erro no sinal de medição e a resposta da $\mathrm{RNAAM}_{3} ;$ o gráfico inferior esquerdo mostra o resíduo nos sinais, e o gráfico à direita mostra a resposta do MLD. Como pode ser observado, o modelo detecta corretamente a falha. O MLD foi testado, também, para um desvio de $0,2^{\circ} \mathrm{F}$ por minuto no Sensor 1/Grupo 1 (Temperatura de escape no cilindro 1LB) a partir do instante 500. A probabilidade de falso alarme neste sensor, que apresenta uma faixa de medição distinta da do primeiro caso, foi fixada em 0,05 , e a de ausência de alarme, em 0,01 . A Figura 14 mostra o desvio no sinal de medição e a resposta da RNAAM 1 , o resíduo nos sinais e a saída do MLD. Como se observa, o modelo responde corretamente no momento em que o desvio ocorre.

\section{CONCLUSÕES}

Este artigo apresentou em detalhes a proposta de um modelo de monitoramento on-line para sensores industriais baseado no uso de redes neurais auto-associativas. O modelo propõe o agrupamento de sensores com graus de correlação similares em diferentes grupos e atribui a cada um destes uma rede, que efetua a auto-correção das medidas de sensores com falhas. Com o treinamento modificado proposto neste artigo, o modelo generaliza e reconstrói sinais quando o sistema de monitoramento detecta falhas simultâneas em distintos sensores de um mesmo grupo. Na avaliação, utilizaram-se medidas de três grupos de sensores instalados num motor real. As três RNAAMs mostraram-se capazes de realizar auto-correções com erros MAPE inferiores a 2\%, mesmo quando a medida do sensor apresentava erros de até 100 unidades.

Ressalte-se que o treinamento das redes neurais envolve um alto custo computacional. No entanto, depois de treinadas, as RNAAMs podem ser utilizadas on-line na correção de falhas de medidas de sensores, com um baixo nível de erro.

O Modelo de Lógica de Decisão, baseado na estatística SPRT, alerta o especialista quando os sensores apresentam falhas e devem ser considerados para manutenção. Nos testes, os modelos responderam satisfatoriamente a simulações de falha do tipo offset, ruído e desvio. Finalmente, é possível afirmar que o modelo gera prioridades na manutenção dos sensores e, assim, reduz os custos associados.

\section{REFERÊNCIAS}

Afonso, P., Ferreira, J. and Castro, J. (1998). Sensor fault detection and identification in a pilot plant under process control, Chemical Engineering Research and Design 76(4): 490-498. 

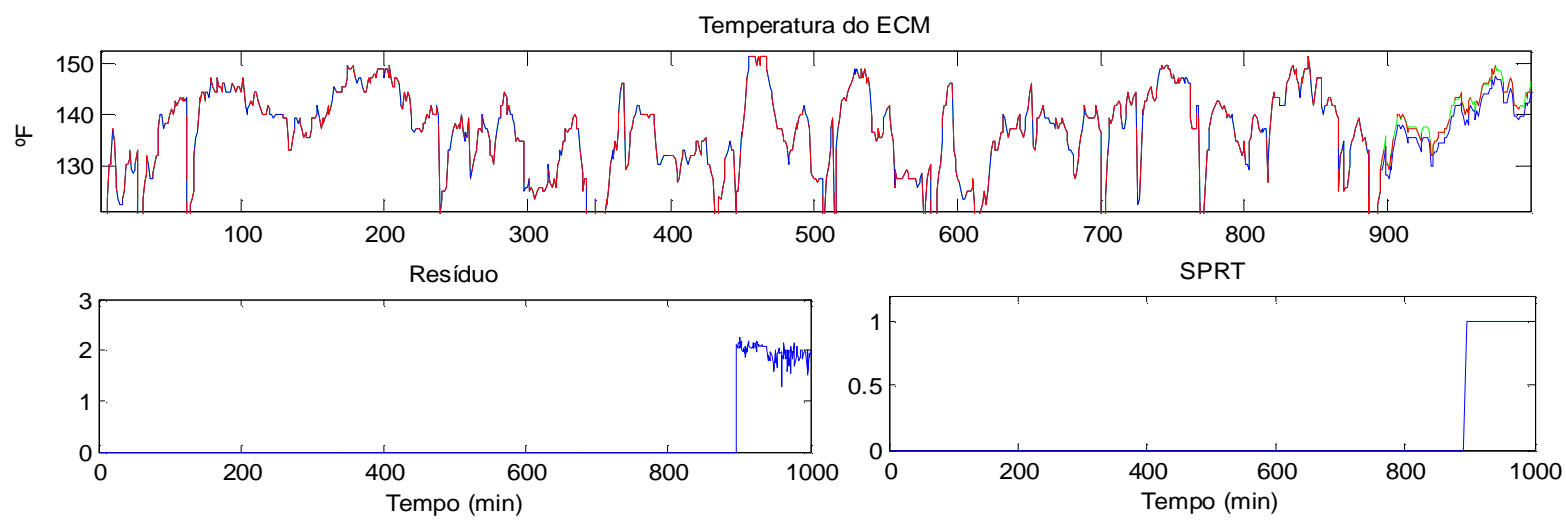

Figura 13: Resposta do MLD frente ao erro no sensor $9 /$ Grupo $3\left(\right.$ offset $=2^{\circ} \mathrm{F}$ ).
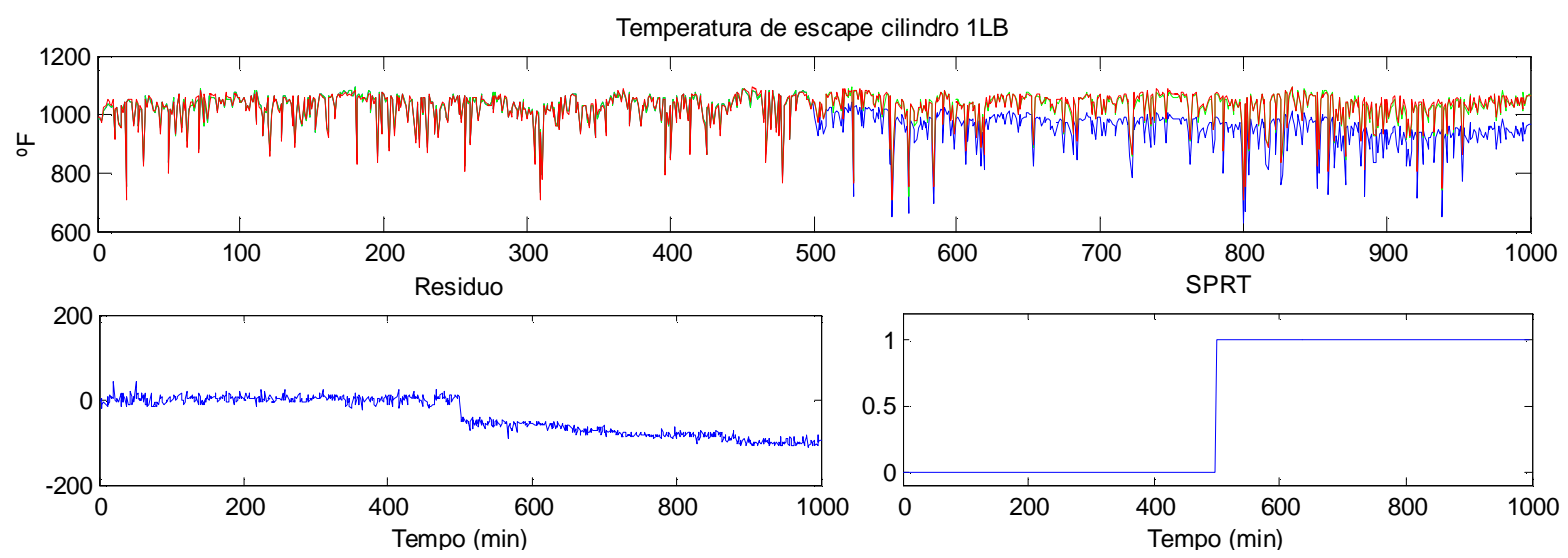

Figura 14: Resposta do MLD frente ao desvio no sensor $1 /$ Grupo 1 ( $5^{\circ} \mathrm{F}$ por minuto).

Antory, D., Irwin, G. W., Kruger, U. and McCullough, G. (2008). Improved process monitoring using nonlinear principal component models, International Journal of Intelligent Systems 23(5): 520-544.

Böhme, T., Fletcher, I. and Cox, C. (1999). Reliable neuro self-tuning control using autoassociative neural networks for the water treatment, $E \&$ I Elektrotechnik und Informationstechnik 116: 375-389.

Bueno, I. (2006). Utilização de redes neurais artificias na monitoração e detecção de falhas em sensores do reator iea-rl, Master's thesis, IPEN/USP.

Cuenca, W. M., Seixas, J. M. and Levy, A. F. (2004). A análise de componentes principais para identificar descargas parciais em transformadores de potência, Anais do Simpósio Brasileiro de Redes Neurais.

de Miguel, L. J. and Blázquez, L. F. (2005). Fuzzy logicbased decision-making for fault diagnosis in a dc motor, Engineering Applications of Artificial Intelligence 18(4): 423-450.
Eyng, E. (2008). Controle Feed Forward-Feedback Aplicado as Colunas de Absorção do Processo de Produção de Etanol por Fermentação, PhD thesis, Faculdade de Engenharia Química - UNICAMP.

Fantoni, P., Hoffmann, M., Shankar, R. and Davis, E. (2003). On-line monitoring of instrument channel performance in nuclear power plant using peano, Progress in $\mathrm{Nu}$ clear Energy 43(1-4): 83-89.

Galotto, L., Bose, B., Leite, L., Pereira Pinto, J., Borges da Silva, L. and Lambert-Torres, G. (2007). Autoassociative neural network based sensor drift compensation in indirect vector controlled drive system, $33 \mathrm{rd}$ Annual Conference of the IEEE Industrial Electronics Society, 2007. IECON 2007., pp. 1009-1014.

Garcia-Alvarez, D., Fuente, M. J., Vega, P. and Sainz, G. (2009). Fault detection and diagnosis using multivariate statistical techniques in a wastewater treatment plant, 7th IFAC International Symposium on Advanced Control of Chemical Processes (2009), pp. 952-957. 
Haykin, S. (1998). Neural Networks - A Comprehensive Foundation, Macmillan College Publishing Company.

Hines, J. W. and Garvey, D. (2007). Process and equipment monitoring methodologies applied to sensor calibration monitoring, Quality and Reliability Engineering International 23(1): 123-135.

Hines, J. W., Uhrig, R. E., Black, C. and Xu, X. (2000). An evaluation of instrument calibration monitoring using artificial neural networks, 8th International Conference on Nuclear Engineering. Proceedings of ICONE 8., Baltimore, MD, USA, pp. 1-9.

Kościelny, J. M. and Syfert, M. (2006). Fuzzy diagnostic reasoning that takes into account the uncertainty of the relation between faults and symptoms, International Journal of Applied Mathematics and Computer Science 16(1): 27-35.

Kramer, M. (1992). Autoassociative neural networks, Computers \& Chemical Engineering 16(4): 313-328.

Kramer, M. A. (1991). Nonlinear principal component analysis using autoassociative neural networks, AIChE Journal 37(2): 233-243.

Marseguerra, M. and Zoia, A. (2005a). The autoassociative neural network in signal analysis: I. the data dimensionality reduction and its geometric interpretation, Annals of Nuclear Energy 32(11): 1191 -1206.

Marseguerra, M. and Zoia, A. (2005b). The autoassociative neural network in signal analysis: Ii. application to online monitoring of a simulated bwr component, Annals of Nuclear Energy 32(11): 1207-1223.

Marseguerra, M. and Zoia, A. (2006). The autoassociative neural network in signal analysis: Iii. enhancing the reliability of a nn with application to a bwr, Annals of Nuclear Energy 33(6): 475-489.

Mendel, J. (1995). Fuzzy logic systems for engineering: a tutorial, Proceedings of the IEEE 83(3): 345-377.

Monsef, W. A., Saraya, S. and Areed, F. (2007). Design of a neural - plc controller for industrial plant, International Conference on Machine Learning, Models, Technologies and Applications (MLMTA), Las Vegas, Nevada, USA, pp. 25-28.

Najafi, M., Gulp, C. and Langari, R. (2004). Enhanced autoassociative neural networks for sensor diagnostics (eaann), IEEE International Conference on Fuzzy Systems, 2004. Proceedings., Vol. 1, pp. 453-456.

Oliveira, M., Amaral, J. and Amaral, J. (2009). Algoritmos para auto-ajuste de sensores utilizando redes neurais, 8th Int. Seminar on Elect. Metrology, João Pessoa, PB.
Pereira, J., Carvalho, A., Tatibana, G., Sanches, R., Carrasco, B., Flora, N. and Hines, J. (2006). Uma nova estratégia de modelagem para monitoramento de instrumentos e verificação de calibração usando redes neurais artificiais., 3 o Congresso Brasileiro de P\&D em Petróleo e Gás, Salvador, Brazil.

Qiao, W., Venayagamoorthy, G. and Harley, R. (2009). Missing-sensor-fault-tolerant control for sssc facts device with real-time implementation, IEEE Transactions on Power Delivery 24(2): 740-750.

Reyes, J., Vellasco, M. and Tanscheit, R. (2010). Sistemas de inferência fuzzy para auto-compensação e auto-validação em sensores inteligentes, XVIII Congresso Brasileiro de Automática (CBA 2010), BonitoMS-Brazil.

Sanz, J., Perera, R. and Huerta, C. (2007). Fault diagnosis of rotating machinery based on auto-associative neural networks and wavelet transforms, Journal of Sound and Vibration 302(4-5): 981-999.

Simani, S., Fantuzzi, C. and Beghelli, S. (2000). Diagnosis techniques for sensor faults of industrial processes, IEEE Transactions on Control Systems Technology 8(5): 848-855.

Singh, H. (2004). Development and implementation of an artificially intelligent search algorithm for sensor fault detection using neural networks, Master's thesis, Texas A\&M University.

Theilliol, D., Noura, H. and Ponsart, J.-C. (2002). Fault diagnosis and accommodation of a three-tank system based on analytical redundancy, ISA Transactions 41(3): 365382.

Tian, G., Zhao, Z. and Baines, R. (2000). A fieldbus-based intelligent sensor, Mechatronics 10(8): 835-849.

Torres, R. C., de Seixas, J. M. and Filho, W. S. (2004). Classificação de sinais de sonar passivo utilizando componentes principais não-lineares, Learning and Nonlinear Models 2(2): 60-72.

Upadhyaya, B. and Eryurek, E. (1992). Application of neural networks for sensor validation and plant monitoring, Nuclear Technology 97(2): 170-176.

Wrest, D. J., Hines, J. W. and Uhrig, R. E. (1996). Instrument surveillance and calibration verification through plant wide monitoring using autoassociative neural networks, Proceedings of the 1996 American Nuclear Society International Topical Meeting on Nuclear Plant Instrumentation, Control and Human Machine Interface Technologies (1996), pp. 6-9. 
Xu, X., Hines, J. W. and Uhrig, R. E. (1998). On-line sensor calibration monitoring and fault detection for chemical processes, Maintenance and Reliability Conference (MARCON 98), pp. 12-14. 